\title{
L-asparaginase-based regimens followed by allogeneic hematopoietic stem cell transplantation improve outcomes in aggressive natural killer cell leukemia
}

Ki Sun Jung ${ }^{1,2}$, Su-Hee Cho ${ }^{2}$, Seok Jin Kim ${ }^{1}$, Young Hyeh Ko ${ }^{3}$, Eun-Suk Kang ${ }^{4}$ and Won Seog Kim ${ }^{1 *}$

\begin{abstract}
Aggressive nature killer cell leukemia (ANKL) is a mature NK-T cell lymphoma with worse prognosis, but optimal treatment is unclear. Therefore, we analyzed the efficacy of L-asparaginase-based regimens for ANKL patients. Twenty-one patients who received dexamethasone, methotrexate, ifosfamide, L-asparaginase, and etoposide (SMILE) or etoposide, ifosfamide, dexamethasone, and L-asparaginase (VIDL) chemotherapy at Samsung Medical Center were selected. The overall response rate for all patients was $33 \%(7 / 21) ; 38 \%$ $(5 / 13)$ in SMILE and $40 \%(2 / 5)$ in VIDL, respectively. The median progression-free survival was 3.9 months (95\% Cl 0.0-8.1 months) and median overall survival was 7.0 months (95\% Cl 2.3-11.7 months). Treatment response $(P=0.001)$, hematopoietic stem cell transplantation $(\mathrm{HSCT})(P=0.007)$ and negative conversion of Epstein-Barr virus (EBV) DNA titer after treatment $(P=0.004)$ were significantly associated with survival. Thus, L-asparaginase-based regimens followed by allogeneic HSCT seem to improve the outcome for ANKL patients.
\end{abstract}

Keywords: ANKL, L-asparaginase, Allogeneic HSCT, Response, Survival

\section{Findings}

Aggressive natural killer cell leukemia (ANKL) is a rare lymphoma with aggressive clinical course $[1,2]$. Treatment outcomes are worse and the median survival is less than 2 months [2-4]. However, optimal treatment of ANKL is not determined yet. Because the tumor cells of ANKL produce P-glycoproteins [5, 6], the treatment response to conventional chemotherapies such as cyclophosphamide, doxorubicin, vincristine, and prednisone (CHOP) is ineffective $[4,7,8]$. Instead, methotrexate and $\mathrm{L}$-asparaginase, which are not affected by P-glycoprotein, are effective agents against NK-T cell lymphoma [3, 7, 9]. Therefore, we conducted this study to determine the efficacy of Lasparaginase-based regimes for patients with ANKL.
ANKL patients diagnosed by current World Health Organization classification who received dexamethasone, methotrexate, ifosfamide, L-asparaginase, and etoposide (SMILE) or etoposide, ifosfamide, dexamethasone, and L-asparaginase (VIDL) as first-line or second-line chemotherapy were enrolled from a lymphoma cohort at Samsung Medical Center between January 2008 and May 2015. We conducted in accordance with the Helsinki Declaration in our study, and our study was approved by the institutional review board of Samsung Medical Center. Clinical characteristics at diagnosis were analyzed for enrolled patients. The treatment response was evaluated followed by criteria that reported previous studies $[2,10]$. We defined complete response as (1)

\footnotetext{
* Correspondence: wskimsmc@skku.edu

${ }^{1}$ Division of Hematology-Oncology, Department of Internal Medicine,

Samsung Medical Center, Sungkyunkwan University School of Medicine,

Seoul, Korea

Full list of author information is available at the end of the article
} 
improved laboratory findings (cytopenia and liver function tests) and symptoms (organomegaly and B symptoms), (2) no residual ANKL cells in bone marrow, and (3) no definite abnormalities in imaging including positron emission tomography or computed tomography. We defined partial response as improved laboratory findings, symptoms, and imaging but minimal residual ANKL cells in bone marrow. The Kaplan-Meier method was used for survival analysis, and the log-rank test was used to test differences in survival on univariate analysis.

A total of 21 patients were analyzed in this study. The characteristics for them are presented in Table 1 . Thirteen patients $(62 \%)$ received SMILE as first-line chemotherapy. Of these patients, five patients showed response to SMILE, either complete response $(n=3,23 \%)$ or partial response $(n=2,15 \%)$. Thus, overall response rate (ORR) to SMILE as initial treatment was $38 \%$. Of patients who responded to SMILE, the three complete responders underwent allogeneic hematopoietic stem cell transplantation (HSCT) and were alive. Of the two partial responders, one received mitoxantrone, etoposide and cytarabine induction chemotherapy and is alive without relapse. The other patient underwent autologous HSCT but died from relapse. Five patients (24\%) were given VIDL as initial treatment. Of these patients, two exhibited complete response to VIDL chemotherapy and received allogeneic HSCT. Thus, the ORR to VIDL was $40 \%$. Of three patients (14 \%) treated with SMILE as second-line chemotherapy, one died from sepsis and the other patients showed disease progression despite receiving fulldose SMILE. Thus, the ORR for all patients was $33 \%(7 / 21)$. Treatment outcomes and characteristics of patients who received HSCT are in Additional files 1 and 2.

The median progression-free survival (PFS) was 3.9 months (95 \% CI 0.0-8.1 months, Fig. 1a) and median overall survival (OS) was 7.0 months (95\% CI 2.3-11.7 months, Fig. 1b). When univariate analysis for prognostic factors was performed, patients who received HSCT had a better OS $(P=0.007)$ and PFS $(P=0.042)$ than patients who did not undergo HSCT (Fig. 1c, d).

We also analyzed correlations between Epstein-Barr virus (EBV) DNA titers and survival (Additional file $3)$. EBV DNA titer at diagnosis $(P=0.311)$ and baseline EBV negativity $(P=0.307)$ were not associated with OS. Negativity of EBV DNA titer after treatment was significantly associated with OS $(P=0.004)$. But, no significant difference was seen in OS between the two subgroups according to change pattern of EBV DNA titer during treatment $(P=0.069)$.
Table 1 Baseline patient characteristics $(N=21)$

\begin{tabular}{|c|c|c|}
\hline Characteristics & Number of patients & Percentage \\
\hline Median age, years (range) & $50(16-75)$ & \\
\hline \multicolumn{3}{|l|}{ Sex } \\
\hline Male & 14 & 67 \\
\hline Female & 7 & 33 \\
\hline \multicolumn{3}{|l|}{ PS } \\
\hline $0-1$ & 12 & 57 \\
\hline $2-4$ & 9 & 43 \\
\hline \multicolumn{3}{|l|}{ Initial presentation } \\
\hline Fever & 20 & 95 \\
\hline General weakness & 1 & 5 \\
\hline DOE & 1 & 5 \\
\hline Lt. neck swelling & 1 & 5 \\
\hline B symptom (+) & 20 & 95 \\
\hline Hepatomegaly & 13 & 62 \\
\hline Splenomegaly & 17 & 81 \\
\hline Pancytopenia & 11 & 52 \\
\hline LFT abnormality & 20 & 95 \\
\hline DIC & 9 & 43 \\
\hline AKI & 4 & 19 \\
\hline $\mathrm{LDH}>$ normal & 20 & 95 \\
\hline Stage IV & 21 & 100 \\
\hline \multicolumn{3}{|l|}{ IPI score } \\
\hline Low & 1 & 5 \\
\hline Low intermediate & 7 & 33 \\
\hline High intermediate & 7 & 33 \\
\hline High & 6 & 29 \\
\hline Nodal involvement & 7 & 33 \\
\hline Extranodal involvement & 7 & 33 \\
\hline EBV ISH (+) & 17 & 81 \\
\hline HLH feature & 16 & 76 \\
\hline PET uptake at diagnosis & 9 & 43 \\
\hline
\end{tabular}

PS performance status, DOE dyspnea on exertion, LFT liver function test, DIC disseminated intravascular coagulopathy, AKI acute kidney injury, LDH lactate dehydrogenase, IPI international prognosis index, EBV ISH Epstein-Barr virus in situ hybridization, HLH hemophagocytic lymphohistiocytosis

Clinical treatment response showed significant association with OS $(P<0.001)$.

In conclusion, early diagnosis and the use of an Lasparaginase-based regimen at initial diagnosis had promising efficacy for patients with ANKL. Also, allogeneic HSCT for responders to an L-asparaginase-based regimen might improve treatment outcomes for patients with ANKL. 


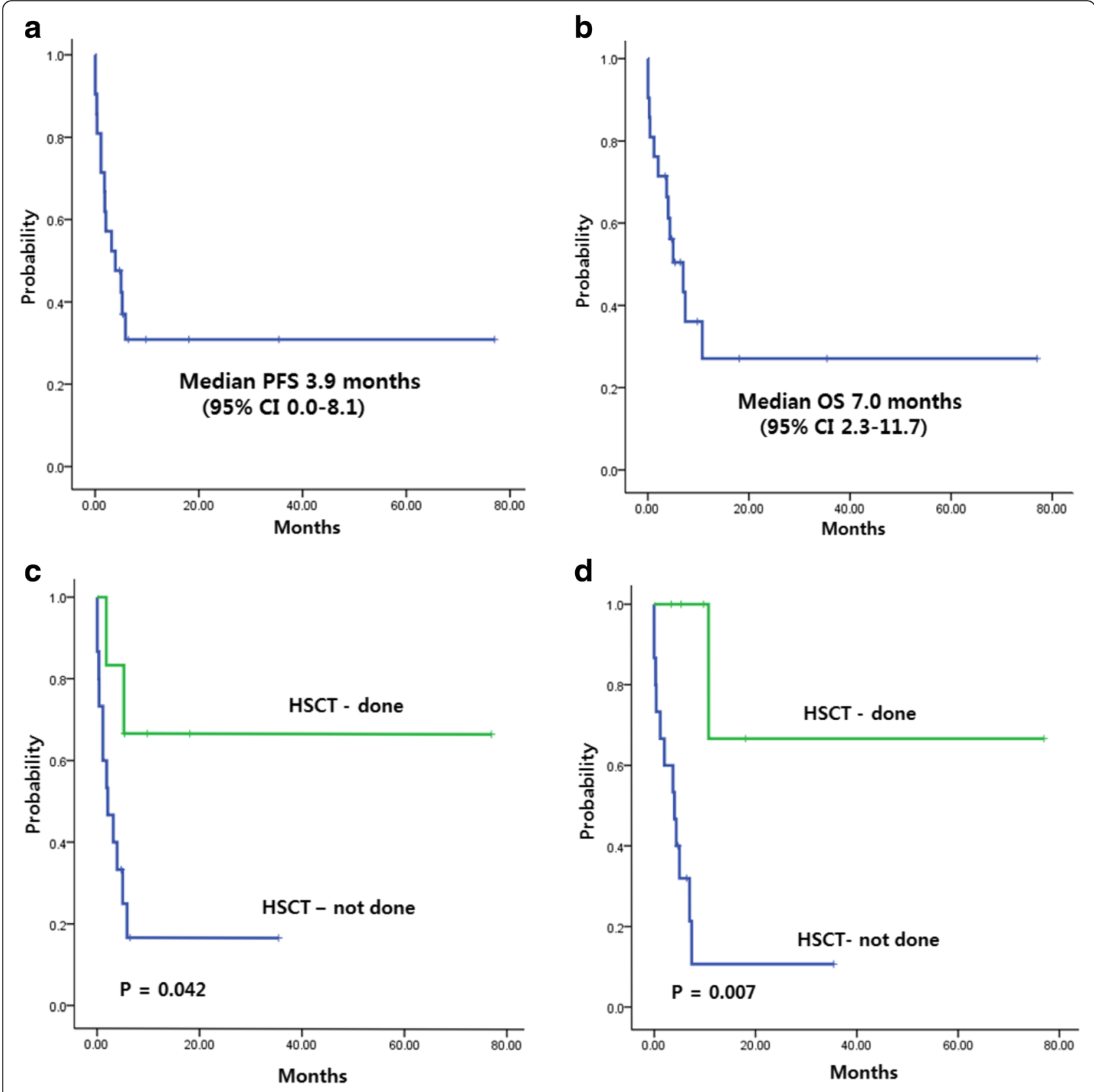

Fig 1 a Progression-free survival and $\mathbf{b}$ overall survival of 21 patients. $\mathbf{c}$, d PFS and OS for patients who underwent HSCT and those who did not receive HSCT

\section{Additional files}

Additional file 1: Summary of patient response and chemotherapy outcomes (A) SMILE as first-line chemotherapy. (A) Thirteen patients (62\%) received SMILE as first-line chemotherapy and 5 patients showed treatmentresponse. Thus, ORR was $38 \%$. (B) Five patients (24\%) underwent VIDL as first-line chemotherapy and 2 patients showed treatment response. Thus, ORR was $40 \%$. (C) Three patients (14\%) treated with SMILE as second-line chemotherapy but no responder showed. (DOCX 97 kb)
Additional file 2: Summary of patients who underwent HSCT after SMILE or VIDL chemotherapy. One patient underwent autologous HSCT and 7 patients received allogeneic HSCT. No engraftment failure or treatment-related mortality occurred. But, 2 patients died from disease relapse (Autologous; 1, Allogeneic; 1). (DOCX 17 kb)

Additional file 3: Survival according to EBV DNA titer and treatment response (A) Survival according to baseline EBV DNA. (A) Baseline EBV negativity did not affect OS ( $p=0.307)$. (B) Negativity EBV DNA after 
treatment was significantly associated with OS $(p=0.004)$. (C) Change pattern (decreasing vs. increasing) of EBV DNA titer during treatment was not significantly associated with OS $(p=0.069)$. (D) Clinical treatment response showed significant association with OS $(p<0.001)$. (DOC 103 $\mathrm{kb})$

\section{Abbreviations}

ANKL: aggressive nature killer cell leukemia; CHOP: cyclophosphamide, doxorubicin, vincristine, and prednisone; EBV: Epstein-Barr virus; HSCT: hematopoietic stem cell transplantation; ORR: overall response rate; OS: overall survival; PFS: progression-free survival; SMILE: dexamethasone, methotrexate, ifosfamide, L-asparaginase, and etoposide; VIDL: etoposide ifosfamide, dexamethasone, and L-asparaginase.

\section{Competing interests}

The authors declare that they have no competing interests.

\section{Authors' contributions}

KSJ, SHC, YHK, and ESK participated in the design of the study and performed the statistical analysis. KSJ, SHC, and SJK wrote the manuscript; SJK and WSK conceived of the study and participated in its design and coordination. All authors read and approved the final manuscript.

\section{Author details}

'Division of Hematology-Oncology, Department of Internal Medicine, Samsung Medical Center, Sungkyunkwan University School of Medicine, Seoul, Korea. ${ }^{2}$ Division of Hematology-Oncology, Department of Internal Medicine, Pusan National University Yangsan Hospital, Pusan National University School of Medicine, Yangsan, Korea. ${ }^{3}$ Department of Pathology, Samsung Medical Center, Sungkyunkwan University School of Medicine, Seoul, Korea. ${ }^{4}$ Department of Laboratory Medicine and Genetics, Samsung Medical Center, Sungkyunkwan University School of Medicine, Seoul, Korea.

Received: 5 April 2016 Accepted: 11 April 2016

\section{Published online: 18 April 2016}

\section{References}

1. Oshimi K. Progress in understanding and managing natural killer-cell malignancies. Br J Haematol. 2007;139:532-44.

2. Song SY, Kim WS, Ko YH, Kim K, Lee MH, Park K. Aggressive natural killer cell leukemia: clinical features and treatment outcome. Haematologica. 2002;87: 1343-5.

3. Ishida F, Ko YH, Kim WS, Suzumiya J, Isobe Y, Oshimi K, et al. Aggressive natural killer cell leukemia: therapeutic potential of L-asparaginase and allogeneic hematopoietic stem cell transplantation. Cancer Sci. 2012;103:1079-83.

4. Suzuki R, Suzumiya J, Nakamura S, Aoki S, Notoya A, Ozaki S, et al. Aggressive natural killer-cell leukemia revisited: large granular lymphocyte leukemia of cytotoxic NK cells. Leukemia. 2004;18:763-70.

5. Yamaguchi M, Kita K, Miwa H, Nishii K, Oka K, Ohno T, et al. Frequent expression of P-glycoprotein/MDR1 by nasal T-cell lymphoma cells. Cancer. 1995;76:2351-6.

6. Egashira M, Kawamata N, Sugimoto K, Kaneko T, Oshimi K. P-glycoprotein expression on normal and abnormally expanded natural killer cells and inhibition of P-glycoprotein function by cyclosporin A and its analogue, PSC833. Blood. 1999;93:599-606.

7. Suzuki R. Treatment of advanced extranodal NKT cell lymphoma, nasal-type and aggressive NK-cell leukemia. Int J Hematol. 2010;92:697-701.

8. LaPorte J, Morris L, Koepke J. Long complete remission achieved with the combination therapy of cisplatin and gemcitabine in a patient with aggressive natural killer cell leukemia. Case Rep Hematol. 2015;2015:715615.

9. Tse E, Kwong YL. How I treat NK/T-cell lymphomas. Blood. 2013:121:4997-5005.

10. Ito T, Makishima H, Nakazawa H, Kobayashi $H$, Shimodaira S, Nakazawa Y, et al. Promising approach for aggressive NK cell leukaemia with allogeneic haematopoietic cell transplantation. Eur J Haematol. 2008;81:107-11.

\section{Submit your next manuscript to BioMed Central and we will help you at every step:}

- We accept pre-submission inquiries

- Our selector tool helps you to find the most relevant journal

- We provide round the clock customer support

- Convenient online submission

- Thorough peer review

- Inclusion in PubMed and all major indexing services

- Maximum visibility for your research

Submit your manuscript at www.biomedcentral.com/submit 